\title{
Optimal Laser Pulse Repetition Rate for Fast In Vivo Imaging using Multiphoton Light-sheet Microscopy
}

Vincent Maioli, Antoine Boniface, Pierre Mahou, Júlia Ferrer Ortas, Lamiae Abdeladim, Emmanuel Beaurepaire, Willy Supatto Laboratory for Optics and Biosciences, Ecole Polytechnique, CNRS, INSERM, Institut Polytechnique de Paris, France

\section{Abstract Text}

Light-sheet microscopy has demonstrated for many years its ability for fast in vivo imaging of delicate samples. The orthogonal arrangement of excitation and detection reduces the photodamage compared to collinear microscopy techniques such as confocal imaging by limiting out-of-focus excitation. This orthogonal geometry exhibits additional benefits in the case of two-photon excited fluorescence with pulsed lasers. Indeed, the low focusing used to generate the light sheet implies lower peak intensities at the sample compared to other fast multiphoton microscopy techniques, mitigating highly nonlinear photodamage without compromising signal, axial resolution nor optical sectioning. In addition, compared to multifocal multiphoton microscopy, the parallelization of the excitation is obtained without splitting the beam, which is critical in the case of a nonlinear optical process and results in lower illumination mean power required. Together, fast imaging is achieved using lower peak intensity and mean power compared to other strategies for fast multiphoton microscopy thanks to this orthogonal arrangement. Does it result in a different photodamage regime compared to point-scanning microscopy and a different strategy to balance fluorescence signal and photodamage? Is $80 \mathrm{MHz}$ laser repetition rate commonly used multiphoton microscopy adapted to take full advantage of light-sheet microscopy? In this study, we investigated these unexplored questions. We aimed at quantifying the influence of laser parameters (pulse frequency, wavelength, and mean power) on both nonlinear signals and photoperturbations during in vivo imaging using multiphoton light-sheet microscopy. To 
this end, we developed a systematic experimental workflow using the heartbeat rate of zebrafish embryos as a reporter of linear and nonlinear disruptions. We then quantified linear effects such as heating and established the scaling laws of nonlinear photodamage. This study demonstrates thermal effects potentially due to water absorption dominates photoperturbation in current implementations of two-photon light-sheet microscopes using $80 \mathrm{MHz}$ femtosecond laser sources, unlike in the case of other multiphoton techniques. As a result, we show an order-of-magnitude increase in signal can be obtained at constant heating effect and without reaching nonlinear photodamage threshold using lower laser pulse repetition $(\sim 10 \mathrm{MHz})$ [1]. Applying this new optimum to live imaging, we could capture cardiac valve motion at 500 frames per second using two-photon excited fluorescence. Such significant improvement using optimal laser duty cycle opens new opportunities for fast and deep in vivo imaging using multiphoton light-sheet microscopy. 